\title{
Screening and verification of proteins that interact with HSPC238
}

\author{
JIA-YU TAN $^{1}$, JING-LIN CHEN $^{2}$, XIANG HUANG $^{2}$ and CHUN-LEI YUAN ${ }^{3}$ \\ ${ }^{1}$ Central Intensive Care Unit, ${ }^{2}$ Prenatal Diagnosis Center, ${ }^{3}$ Department of Laboratory Medicine, \\ Bo'ai Hospital of Zhongshan City Affiliated with Southern Medical University, Zhongshan 528403, P.R. China
}

Received June 24, 2015; Accepted July 14, 2015

DOI: $10.3892 /$ or.2015.4289

\begin{abstract}
HSPC238 is a recently identified tumor suppressor and demonstrates ubiquitin ligase E3 enzyme activity. HSPC238 was found to be significantly downregulated in human hepatocellular carcinoma (HCC) in vivo and to inhibit the proliferation and invasion of hepatoma cells in vitro; however, the underlying molecular mechanism is largely unknown. In the present study, we screened for and identified proteins that physically interact with HSPC238. A bait vector for yeast two-hybrid was constructed with human HSPC238 gene cDNA. Yeast two-hybrid screening was performed using a human fetal liver cDNA library. Multiple reporter gene assays, DNA sequencing and BLAST comparison analysis were performed on positive clones. Protein interaction of screened candidates with HSPC238 was further validated by confocal microscopy, co-immunoprecipitation and pulldown assays. Yeast two-hybrid screening demonstrated 124 positive clones. Multiple reporter gene assays with $L a c Z$, $H I S$ and $A D E 2$ selective media identified 12 genes. Further co-localization, co-immunoprecipitation and pull-down assays demonstrated that HMOX1, RPS27A, ubiquitinB and MT2A interacted with HSPC238. These four proteins are involved in tumor development and progression, and are associated with the ubiquitin-proteasome pathway. Our results suggest that HSPC238 may play a tumor suppressor role and interact with these proteins via the ubiquitin-proteasome pathway. The identification and validation of proteins interacting with HSP238 may lead to the discovery of novel mechanisms through which HSPC238 suppresses tumorigenesis in human hepatocellular carcinoma.
\end{abstract}

\section{Introduction}

HSPC238, also known as RNF181 (ring finger protein 181), is a tumor suppressor gene located at chromosome 2p11.2 (1).

Correspondence to: Professor Xiang Huang, Prenatal Diagnosis Center, Bo'ai Hospital of Zhongshan City Affiliated with Southern Medical University, Zhongshan 528403, P.R. China

E-mail: 340382761@qq.com; xiaotaoqi1998@163.com

Key words: HSPC238, yeast two-hybrid, protein interaction, hepatocellular carcinoma
It encodes a 153 amino acid protein that contains a RING finger domain in its first 40 to 60 amino acid residues. It has been reported that the HSPC238 protein has ubiquitin ligase E3 enzyme activity (2). In a previous study, our group found that HSPC238 was significantly downregulated in HCC when compared with adjacent tissues. Further studies showed that HSPC 238 could significantly inhibit the proliferation and invasion of hepatoma cells in vivo and in vitro, and that it played a tumor suppressor role in the development of HCC (3). Previous studies also showed that HSPC238 inhibited the growth of HepG2 and SMMC7721 cells through the ERK/MAPK pathway, but the underlying molecular mechanism is unclear.

The zinc finger proteins are a class of important transcription factors that are deregulated in many human diseases, including cancer (4). Moreover, the zinc finger domain may be involved in many biological processes via binding of $\mathrm{Zn}^{2+}$ and interactions with other proteins. However, it is unclear with which proteins HSPC238 interacts. In the present study, to provide a foundation for further subsequent study of HSPC238's tumor suppressor function, we performed a yeast two-hybrid assay to identify the proteins that interact with the HSPC238, followed by validation with confocal microscopy, co-immunoprecipitation and pull-down assays.

\section{Materials and methods}

Reagents and cells. The yeast two-hybrid system and the human fetal liver cDNA library were purchased from Clontech Laboratories, Inc., (Mountain View, CA, USA). 293T, HepG2 and SMMC7721 cell lines were purchased from the American Type Culture Collection (ATCC; Manassas, VA, USA). Cells were grown in RPMI-1640 medium (Gibco, Life Technologies, Grand Island, NY, USA) containing $10 \%$ fetal bovine serum (FBS), $100 \mathrm{U} / \mathrm{ml}$ penicillin and $100 \mu \mathrm{g} / \mathrm{ml}$ streptomycin and were incubated at $37^{\circ} \mathrm{C}$ in a $5 \% \mathrm{CO}_{2}$ atmosphere. Lipofectimine 2000 transfection reagents, and the plasmids pGBKT7, pGADT7 and pCDNA3.1 (+) were purchased from Life Technologies. DNA gel purification kits and a small plasmid extraction kits were purchased from Axygen (Tewksbury, MA, USA). Anti-HSPC238 rabbit antibody was obtained from Proteintech Group Inc. (Chicago, IL, USA); anti-HMOX1, antiMT2A, anti-RPS27A and anti-ubiquitin B (UBB) antibodies were purchased from Abclonal (Cambridge, MA, USA); and anti-HIS mouse antibody and anti-Flag mouse antibody were purchased from Zoonbio Tech Co., Ltd. (Nanjing, China). The secondary CY3-labeled goat anti-rabbit and FITC-labeled 
goat anti-mouse antibodies were purchased from Rockland Immunochemicals Inc. (Gilbertsville, PA, USA).

Yeast two-hybrid assay. The ORF of HSPC238 gene was amplified by PCR and subcloned into the yeast two-hybrid bait vector pGBKT7 to get vector pGBKT7-HSPC238. The vectors pGBKT7-HSPC238 and pGADT7 were co-transformed into the yeast strain AH109 in SD-Trp-Leu medium. Three reporter genes ( $L a c Z, H I S$ and $A D E 2$ ) were used to detect self-activation of yeast transformants (pGBKT7-HSPC238 + pGADT7). Transformants with no self-activation of the three reporter genes were used for subsequent library screening. Matchmaker Human Fetal Liver Library plasmids (cat. no. 638805; Clontech) were transformed into yeast AH109 that had been co-transformed with pGBKT7-HSPC238 and pGADT7. The transformants were plated on SD-3 + 3AT agar medium and incubated for one to two weeks. One hundred and twenty-four transformants were further tested with the three reporter genes ( $L a c Z, H I S$ and $A D E 2)$. Positive clone strains were inoculated in liquid SD-Leu medium. Yeast plasmids were extracted and further transformed into E. coli Top10 fresh competent cells for amplification. Following extraction from E. coli, the plasmids were subjected to DNA sequencing and BLAST alignment analysis.

Confocal laser location detection. HSPC238 and target genes (HMOX1, RPS27A, UBB and MT2A) were cloned into pcDNA3.1(+) to get the recombinant plasmids HSPC238pcDNA3.1(+), HMOX1-pcDNA3.1(+), UBB-pcDNA3.1(+), RPS27A-pcDNA3.1(+) and MT2A-pcDNA3.1(+). 293T or SMMC7721 cells were transformed with the HSPC238pcDNA3.1(+) plasmid together with pcDNA3.1(+)-target plasmids. Colocalization of HSPC238 with the four proteins in 293T and SMMC7721 cells was observed by confocal microscopy using antibodies against the recombinant target protein and HSPC238.

Co-immunoprecipitation. Cells were washed with prechilled PBS twice, re-suspended in cold RIPA lysis buffer (20 mmol/1 Tris-HCl, pH 7.5; $150 \mathrm{mmol} / 1 \mathrm{NaCl} ; 1 \mathrm{mmol} / 1$ $\mathrm{Na}_{2}$ EDTA; $1 \mathrm{mmol} / 1$ EGTA; $1 \%$ Triton; $2.5 \mathrm{mmol} / 1$ sodium pyrophosphate; $1 \mathrm{mmol} / 1 \beta$-glycerophosphate; $1 \mathrm{mmol} / 1$ $\mathrm{Na}_{3} \mathrm{VO}_{4} ; 1 \mathrm{mg} / \mathrm{ml}$ leupeptin; and $1 \mathrm{mmol} / \mathrm{l}$ phenylmethylsulfonylfluoride) and lysed on ice for $15 \mathrm{~min}$ at $4^{\circ} \mathrm{C}$. Protein A/G-agarose beads were washed twice with PBS and diluted to $50 \%$ protein $\mathrm{A} / \mathrm{G}$ agarose in PBS. After centrifuging at $14,000 \mathrm{x} g$ and $4^{\circ} \mathrm{C}$ for $15 \mathrm{~min}$, the cell lysate supernatant was immediately transferred to new tubes and added to the $50 \%$ protein A/G agarose at a ratio of $100 \mu \mathrm{l} / 1 \mathrm{ml}$ sample solution. After shaking on a horizontal shaker at $4^{\circ} \mathrm{C}$ for $10 \mathrm{~min}$, the mixture was centrifuged at $14,000 \mathrm{x} \mathrm{g}$ for $15 \mathrm{~min}$ at $4^{\circ} \mathrm{C}$. The supernatant was transferred to new tubes and the concentration of the total protein was determined with a BCA assay. Total protein $(500 \mu \mathrm{g})$ was mixed with $10 \mu \mathrm{g}$ primary antibody in $500 \mu \mathrm{l}$ of solution and shaken on a rotating shaker at $4^{\circ} \mathrm{C}$ overnight. The next day, the mixture was centrifuged at $14,000 \mathrm{x} g$ for $5 \mathrm{sec}$. The pellet was washed with pre-chilled PBS three times, re-suspended in $15 \mu 12 \mathrm{X}$ SDS sample buffer and boiled at $100^{\circ} \mathrm{C}$ for $5 \mathrm{~min}$. The sample was then subjected to western blot analysis.
Pull-down assay. The purified 6xHis-tagged HSPC238 protein (6-10 mg) in $100 \mu \mathrm{l}$ buffer B [50 mM sodium phosphate buffer, $\mathrm{pH} 7.5,100 \mathrm{mM} \mathrm{NaCl}, 5 \mathrm{mM} \mathrm{MgCl}{ }_{2}, 2 \mathrm{mM} \beta$-mercaptoethanol and $100 \mu \mathrm{g}$ bovine serum albumin (BSA)/ml] was incubated with $1 \mathrm{ml}$ of a $50 \%$ slurry of Ni-NTA-agarose resin (Qiagen Inc., Valencia, CA, USA) that was pre-equilibrated in buffer B. After 90-min incubation at room temperature, the beads were washed three times with $400 \mu \mathrm{l}$ buffer B and twice with $400 \mu \mathrm{l}$ buffer B with $50 \mathrm{mM}$ imidazole. After an additional wash with $100 \mu \mathrm{l}$ buffer B with $50 \mathrm{mM}$ imidazole, proteins bound to the beads were released by adding $100 \mu \mathrm{l}$ buffer B with $350 \mathrm{mM}$ imidazole. The presence of HSPC238 in different fractions was analyzed by western blotting.

Western blotting. Equal amounts of protein from each sample were denatured in SDS sample buffer and separated on a $12 \%$ SDS polyacrylamide gel. Proteins were transferred to polyvinylidene difluoride (PVDF) membranes in buffer containing $25 \mathrm{mM}$ Tris, $192 \mathrm{mM}$ glycine and 20\% methanol. Membranes were blocked with Tris-buffered saline (TBS; $137 \mathrm{mM} \mathrm{NaCl}$, $20 \mathrm{mM}$ Tris- $\mathrm{HCl}, \mathrm{pH} 7.5)$ containing $0.05 \%$ Tween-20 and 5\% dried milk powder, incubated first with primary antibodies and then with horseradish peroxidase-conjugated secondary antibodies. Signals were developed with the enhanced chemiluminescence detection system (Pierce, Thermo Fischer Scientific, Bonn, Germany).

\section{Results}

Yeast two-hybrid screening results. To identify proteins that potentially physically interact with HSPC238, we performed a yeast two-hybrid screen with HSPC238 as bait against Matchmaker Human Fetal Liver Library. One hundred and twenty-four positive clones were picked from the first screen and tested with $L a c Z, H I S$ and $A D E 2$ selective media. Twelve genes were found that potentially interacted with HSPC238 (Table I) after sequencing plasmid DNA from clones that passed through successive screenings. After reviewing the literature, we finally selected $H M O X 1, M T 2 A, R P S 27 A$ and $U B B$ for further validation.

HMOX1, MT2A, RPS27A and UBB localize in the cytoplasm and co-localize with HSPC238. We transfected 293T or SMMC7721 cells with pcDNA3.1-HSPC238-Flag together with either pcDNA3.1-HMOX1-6xHis, pcDNA3.1s-RPS27A6xHis, pcDNA3.1-UBB-6xHis or pcDNA3.1-MT2A-6xHis, and determined the co-localization of HSPC238 with the corresponding candidate protein by confocal microscopy. In both 293T and SMMC7721 cells, RPS27A was found to co-localize with HSPC238 (Fig. 1A). Similar co-localization of HSPC238 with MT2A (Fig. 1B), HMOX1 (Fig. 1C) and UBB (Fig. 1D) were observed in both 293T and SMMC7721 cells. Moreover, the results showed that HSPC238, HMOX1, MT2A, RPS27A and UBB all localized in the cytoplasm of 293T and SMMC7721 cells (Fig. 1).

HMOX1, MT2A, RPS27A and UBB can be co-immuno-precipitated with HSPC238. We performed co-immunoprecipitation assays to confirm the physical interaction of HSPC238 with HMOX1, MT2A, RPS27A and UBB. No endogenous Flag or 
Table I. Proteins that potentially interact with HSPC238.

\begin{tabular}{lll}
\hline Name & Accession number & \multicolumn{1}{c}{ Description } \\
\hline UBB & NM_018955.2 & Homo sapiens ubiquitin B (UBB), mRNA \\
MT2A & NM_005953.3 & Homo sapiens metallothionein 2A (MT2A), mRNA \\
HBA1 & NM_000558.3 & Homo sapiens hemoglobin, $\alpha$ 1 (HBA1), mRNA \\
RPS27A & NM_001177413.1 & Homo sapiens ribosomal protein S27a (RPS27A), transcript variant 3, mRNA \\
MT1E & NM_175617.3 & Homo sapiens metallothionein 1E (MT1E), mRNA \\
NDUFB10 & NM_004548.2 & Homo sapiens NADH dehydrogenase (ubiquinone) 1 $\beta$ subcomplex, 10, 22 kDa \\
& & (NDUFB10), nuclear gene encoding mitochondrial protein, mRNA \\
MCM7 & NM_182776.1 & Homo sapiens minichromosome maintenance complex component 7 (MCM7), transcript \\
ALB & NM_000477.5 & Homo sapiens albumin (ALB), mRNA \\
ELL2 & NM_012081.5 & Homo sapiens elongation factor, RNA polymerase II, 2 (ELL2), mRNA \\
MT1H & NM_005951.2 & Homo sapiens metallothionein 1H (MT1H), mRNA \\
RPL5 & NM_000969.3 & Homo sapiens ribosomal protein L5 (RPL5), mRNA \\
FGB & NM_001184741.1 & Homo sapiens fibrinogen $\beta$ chain (FGB), transcript variant 2, mRNA \\
\hline
\end{tabular}
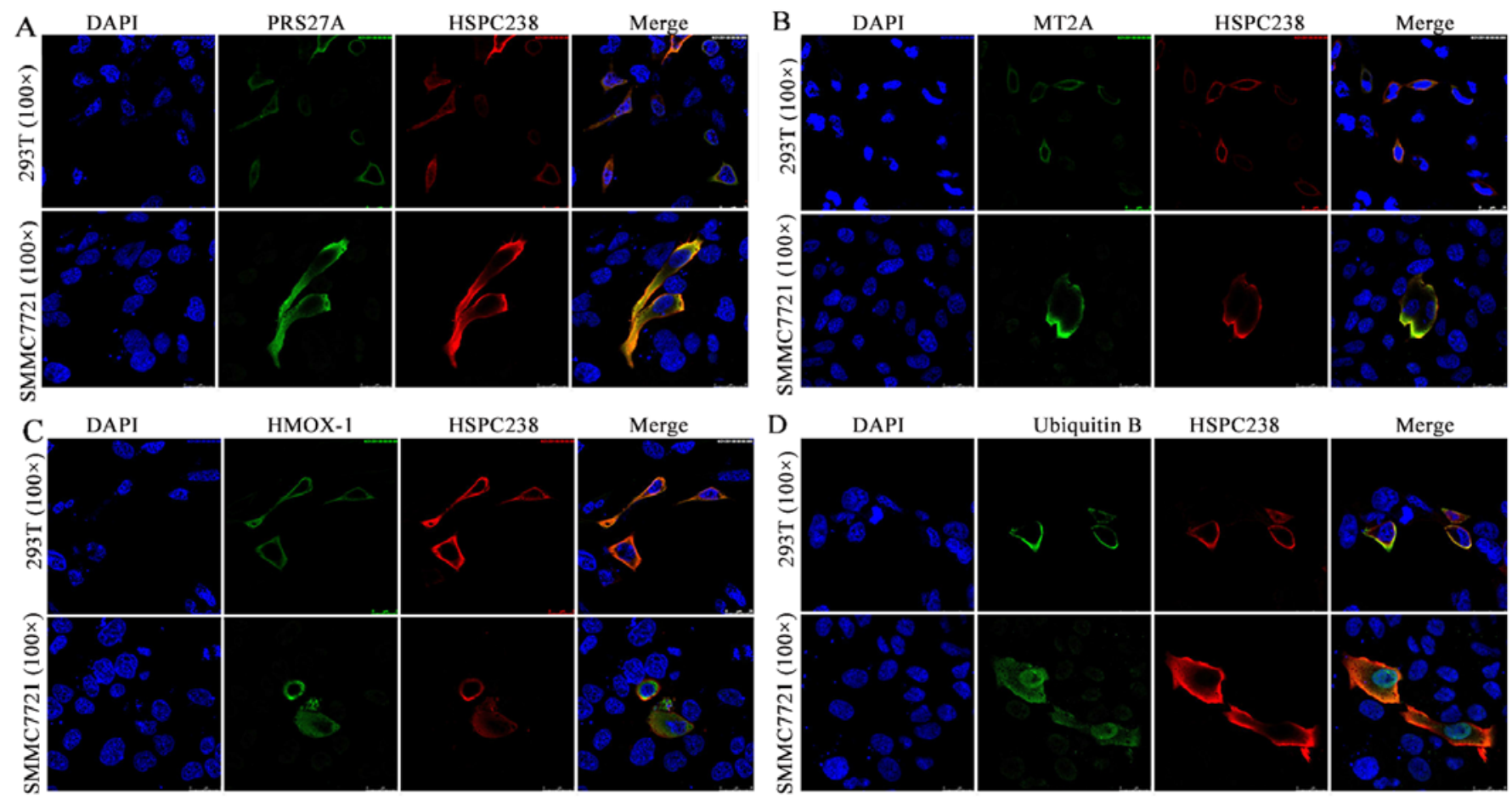

Figure 1. Co-localization of RPS27A/MT2A/HMOX1/UBB and HSPC238 in 293T and SMMC7721 cells. 293T or SMMC7721 cells were co-transfected with pcDNA3.1-HSPC238-Flag together with either pcDNA3.1-HMOX1-6xHis, pcDNA3.1-RPS27A-6xHis, pcDNA3.1-UBB-6xHis or pcDNA3.1-MT2A-6xHis. The co-localization of HSPC238 with (A) RPS27A, (B) MT2A, (C) HMOX1 and (D) UBB, was visualized on a confocal microscope with anti-His and anti-Flag antibodies. The blue fluorescence is the nuclei stained with DAPI, the green fluorescence is the His-tagged target protein and the red fluorescence is Flag-tagged HSPC238.

His was observed in either 293T or SMMC7721 cells, while transfection of pcDNA3.1-HSPC238-Flag (Fig. 2A, left panel) and pcDNA3.1-HMOX1-His (Fig. 2A, right panel) vectors resulted in high levels of Flag and His signals, respectively. Similarly, strong His signals were detected in both cell lines when they were transfected with pcDNA3.1-RPS27A-His, pcDNA3.1-UBB-His or pcDNA3.1-MT2A-His (Fig. 2B). In the HSPC238 single transfection, no specific HMOX1 band was detected with either anti-His or anti-HMOX1 antibody in the IP precipitate or the cell lysates (Fig. 2C). In contrast, following co-transfection of pcDNA3.1-HSPC238-Flag and pcDNA3.1-HMOX1-His, HMOX1 was detected with both anti-His and anti-HMOX1 antibody in both the IP precipitate and the cell lysates (Fig. 2C). Similarly, RPS27A, UBB and 


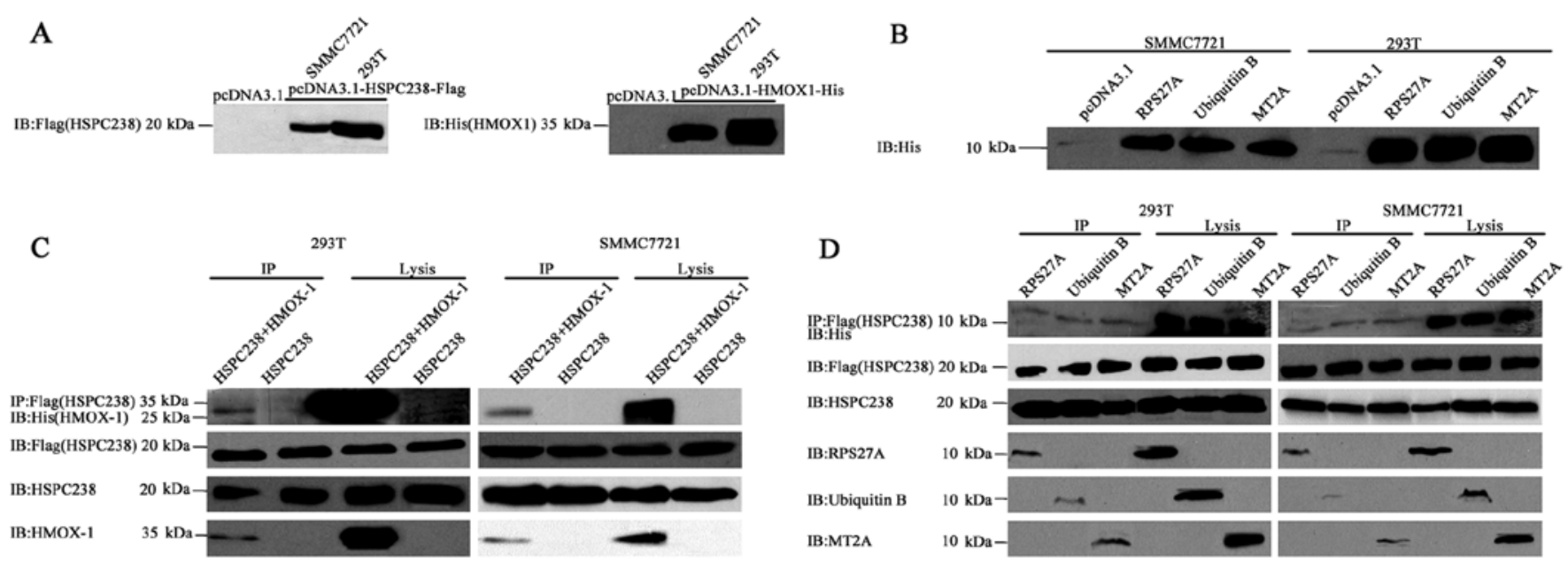

Figure 2. HMOX1, MT2A, RPS27A and UBB co-immunoprecipitate with HSPC238. (A) 293T or SMMC7721 cells were transfected with pcDNA3.1, pcDNA3.1-HSPC238-Flag or pcDNA3.1-HMOX1-6xHis. Total proteins were extracted for immunoblotting of His and Flag tag. (B) 293T or SMMC7721 cells were transfected with pcDNA3.1, pcDNA3.1-RPS27A-6xHis, pcDNA3.1-UBB-6xHis or pcDNA3.1-MT2A-6xHis. Total proteins were extracted for immunoblotting of the His tag. (C) 293T or SMMC7721 cells were co-transfected with pcDNA3.1-HSPC238-Flag, or pcDNA3.1-HSPC238-Flag together with pcDNA3.1-HMOX1-6xHis pcDNA3.1-RPS27A-6xHis, pcDNA3.1-UBB-6xHis or pcDNA3.1-MT2A-6xHis. Total proteins were extracted for co-immunoprecipitation (IP) with anti-Flag antibodies, followed by immunoblotting (IB) with anti-His tag, anti-HSPC238, anti-HMOX-1, anti-RPS27A, anti-UBB and anti-MT2A antibodies. (D) 293T or SMMC7721 cells were co-transfected with pcDNA3.1-HSPC238-Flag or pcDNA3.1-HSPC238-Flag together with pcDNA3.1-HMOX1-6xHis. Total proteins were extracted for co-immunoprecipitation (IP) with anti-Flag antibodies, followed by immunoblotting (IB) with anti-His tag, HSPC238, HMOX-1, RPS27A, UBB and MT2A antibodies.
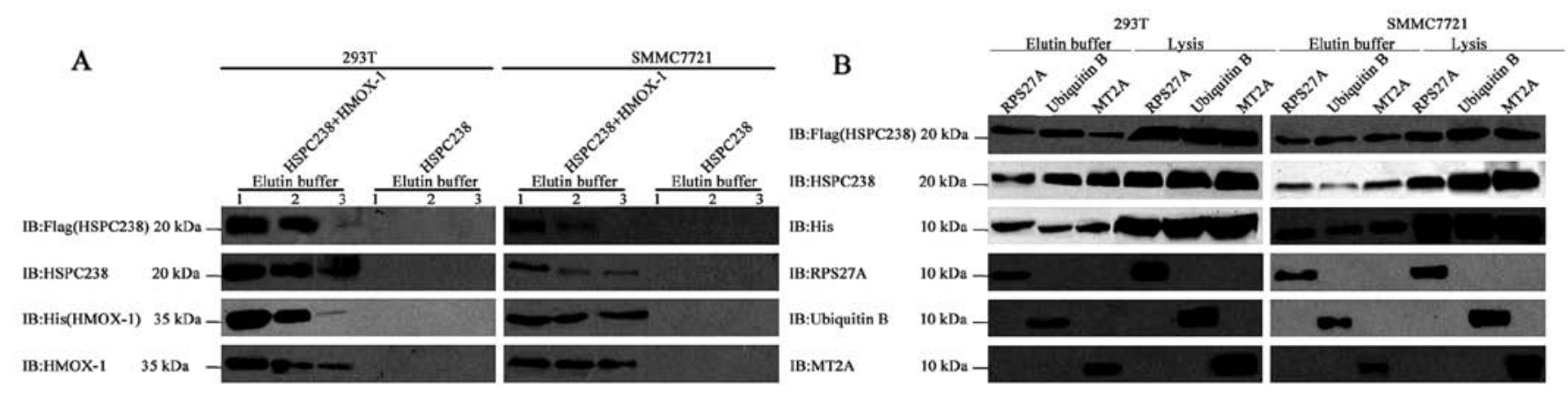

Figure 3. HSPC238 pull-down by HMOX-1, RPS27A, UBB and MT2A. (A) 293T or SMMC7721 cells were transfected with pcDNA3.1-HSPC238-Flag, alone or in combination with pcDNA3.1-HMOX1-6xHis. Total proteins were extracted for simultaneous purification by nickel column. Three eluate samples with higher concentrations were subjected to western blotting. (B) 293T or SMMC7721 cells were transfected with pcDNA3.1-HSPC238-Flag, alone or in combination with pcDNA3.1-RPS27A-6xHis, pcDNA3.1-UBB-6xHis or pcDNA3.1-MT2A-6xHis. Total protein was extracted for simultaneous purification by nickel column. The eluate sample with the highest concentration as well as cell lysates were subjected to western blotting.

MT2A were detected by either anti-His or anti-RPS27A, UBB or MT2A antibody, respectively, in both the IP precipitate and the cell lysates (Fig. 2D). These results indicated that HMOX-1, RPS27A, UBB and MT2A could be co-immunoprecipitated with HSPC238.

HSPC238 can be pulled down by HMOX-1, RPS27A, UBB or $M T 2 A$. We performed protein pull-down assays to examine the interaction of HSPC238 with HMOX1, MT2A, RPS27A and UBB. In the HSPC238 single transfection, no specific HSPC238 bands were detected with either antiFlag or anti-HSPC238 antibody in the eluents (Fig. 3A). In the co-transfection of pcDNA3.1-HSPC238-Flag and pcDNA3.1-HMOX1-His, HMOX1 was detected in the eluent by both anti-His and anti-HMOX1 antibody and HSPC238 was detected by both anti-Flag and anti-HSPC238 antibody. In the co-transfection of pcDNA3.1-HSPC238-
Flag with all three of the other plasmids, RPS27A, UBB and MT2A were detected with anti-His as well as anti-RPS27A, UBB or MT2A antibody, respectively, in both the eluents and the cell lysates. In this co-transfection, HSPC238 was detected by both anti-Flag and anti-HSPC238 antibody in eluents and cell lysates (Fig. 3B). These results indicate that either HMOX-1, RPS27A, UBB or MT2A can pull down HSPC238.

\section{Discussion}

In the present study, we successfully screened 12 proteins that interact with HSPC238 using a yeast two-hybrid assay. Among these proteins, HMOX1, RPS27A, UBB and MT2A were further validated by co-localization, co-immunoprecipitation and pull down assays. Our results provide new insights concerning the molecular functions of HSPC238. 


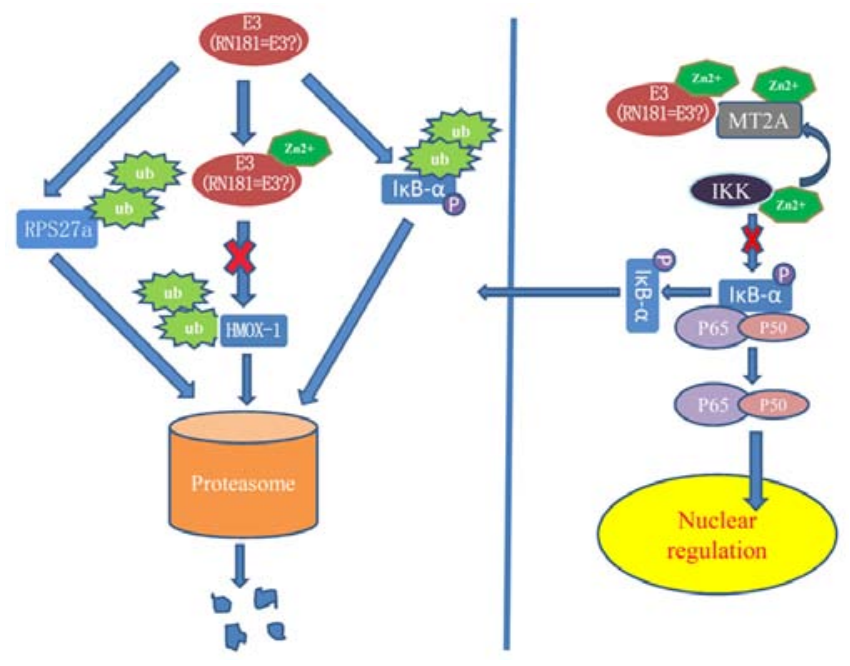

Figure 4. A possible mechanism of HSPC238 interaction with HMOX-1, MT2A, RPS27a and UBB.

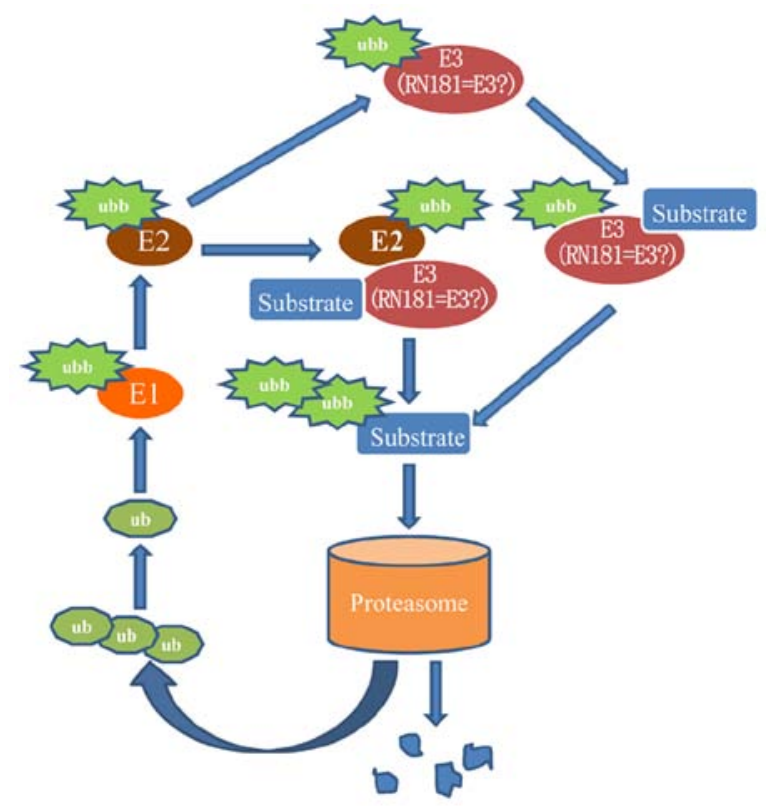

Figure 5. A possible UBB driven mechanism of tumorigenesis suppression through the ubiquitin-proteasome pathway.

HSPC238 is a tumor suppressor gene that is known to have a special RING finger domain (1). The RING finger domain, which is a novel zinc finger protein domain defined by Lovering in 1994, may be involved in many biological processes via binding of $\mathrm{Zn}^{2+}$ and interaction with other proteins. The zinc finger proteins are a class of important transcription factors that are deregulated in many human diseases, including cancers (4). Brophy et al (2) confirmed that HSPC238 has ubiquitin ligase E3 enzyme activity. Because the ubiquitin ligase E3 enzymes are the key enzymes in the ubiquitin-proteasome pathway, HSPC238 may play a tumor suppressor role by promoting the degradation of tumor-promoting proteins or by interfering with the degradation of tumor suppressors.

Previous studies (2-4) showed that HSPC238 inhibited the growth of HepG2 and SMMC7721 cells through the ERK/MAPK pathway, but the underlying molecular mecha- nism remains unclear. In the present study, we demonstrate the physical interaction of HMOX-1, RPS27A, UBB and MT2A with HSPC238 with yeast two-hybrid, confocal laser, co-immunoprecipitation and pull-down techniques. The physical interactions we report here between these proteins may lead to the discovery of novel mechanisms through which HSPC238 regulates the cellular processes.

HSPC238 with RPS27a. RPS27a is a ribosomal protein that plays an important role in protein synthesis. Accumulating evidence indicates that ribosomal proteins have other functions, such as the regulation of DNA replication and repair, mRNA transcription and processing, cell apoptosis, malignant transformation of normal cells and developmental regulation. All of these functions have significance for tumorigenesis and anticancer drug resistance.

Wong et al (5) reported that the RPS27a gene is overexpressed in human colon cancer cells. Ganger et al (6) showed that RPS27a was expressed at low levels in chronic hepatitis cells, but was highly expressed in cirrhotic and hepatocellular carcinoma cells. Similarly, Fatima et al (7) found that RPS27a may be an important factor during the processes of $\mathrm{HB}$ virus-mediated liver cancer. These facts suggest that RPS27a might play a key role in the tumorigenesis of liver cancer. Our previous studies (3) have shown that HSPC238 had ubiquitin ligase E3 activity and significantly inhibited the growth of hepatoma cells in vivo and in vitro. The physical interaction of HSPC238 with RPS27a suggests that HSPC238 may function as a tumor suppressor by promoting the degradation of RPS27a through the ubiquitin-proteasome pathway (Fig. 4).

HSPC238 with HMOX-1. HMOX-1 is an isoform of heme oxygenase, a key heme degradation enzyme that decomposes hemoglobin into carbon monoxide, ferrous ion and biliverdin (8). HMOX-1 plays an important role in the selfprotection of cells; however, HMOX-1 also plays key roles during tumorigenesis through its anti-oxidative and antiapoptotic functions. In a chemical carcinogenesis liver cancer experiment, Cabalero et al (9) found that decreased expression of HMOX-1 is associated with malignant tumor progression. However, HMOX-1 expression in the Kuffer cells of macrophages and tumor nodules in the periphery of necrotic tissue is increased, indicating that HMOX-1 may have a protective role in chronic liver injury and suggesting that HMOX-1 is a potential agent for the treatment of primary liver cancer. Zou et al (10) reported that HMOX-1 inhibited the migration and growth of hepatocellular carcinoma cells by inhibiting the expression of IL-6 in vitro and in vivo. Carbon monoxide generation by heme oxygenase also inhibits the migration of cancer cells by inhibiting the activity of IL- 6 and p38MAPK. These studies indicate that HMOX-1 might prevent the tumorigenesis of liver cancer via a mechanism similar to heme oxygenase. Lin et al (11) reported that HMOX-1 degradation was mediated by a $\mathrm{Zn}^{2+}$ dependent RING-E3 enzyme. $\mathrm{Zn}^{2+}$ chelating agents significantly inhibited the ubiquitination of HMOX-1, whereas ubiquitination was significantly enhanced by $\mathrm{ZnCl}_{2}$. Because HSPC 238 contains a $\mathrm{Zn}^{2+}$ binding zinc finger structure, the interaction of HSPC238 with HMOX-1 suggest that HSPC238 may inhibit tumorigenesis by preventing the ubiquitination and degradation of HMOX-1 (Fig. 4). 
HSPC238 with MT2A. Of all the MT-II protein subtypes, MT-2A is the only one with a known biological effect (12). Not only does MT-2A have anti-radiation, heavy metal detoxification, free radical scavenging, tissue damage repair, trace element balance and anti-aging effects, but it is also associated with cell proliferation, apoptosis, tumor development and drug resistance. MT2A is overexpressed in breast, lung, bladder and ovarian cancer, but downregulated in liver and gastric cancer. It was found that hepatitis B virus infection was associated with downregulation of MT2A expression in hepatoma cells (13). Mao et al (14) reported that exogenous MT1M inhibited the growth of hepatoma cells in vitro and in vivo by reducing TNF- $\alpha$-induced activation of NF- $\kappa \mathrm{B}$. Pan et al (15) showed that MT2A inhibited the activation of the NF- $\kappa B$ pathway by upregulating I $\kappa B$, thereby inhibiting the growth of gastric cancer cells. Similarly, restoring the expression of MT2A in vitro and in vivo could inhibit the growth of gastric cancer cells. Habel et al (16) found that overexpression of MT2A reduced the intracellular activity of zinc ions and the growth kinetics of cells, thereby inhibiting the growth of osteosarcoma cells. In addition, $\mathrm{Zn}^{2+}$ was found to enhance the phosphorylation of $\mathrm{I} \kappa \mathrm{B}$, which in turn promoted the activity of NF-KB (17). Various signals, such as virus infections, lead to the phosphorylation and degradation of IKB via the ubiquitinproteasome pathway. These facts suggest that in the NF- $\kappa B$ pathway, MT2A inhibits the activation of IKK via chelating zinc ions, thereby inhibiting the phosphorylation, ubiquitination and degradation of IкB and, therefore, preventing the activation of NF-кB. HSPC238 can interact with $\mathrm{Zn}^{2+}$, and it may inhibit the activation of NF- $\mathrm{KB}$ by a process similar to that described above. Our present study shows that HSPC238 interacts with MT2A (Fig. 4). We speculate that a HSPC238 and MT2A complex may exert a synergistic antitumor effect.

HSPC238 with UBB. Ubiquitin B is a ubiquitin protein, but its function is largely unknown. Tian et al (18) reported that UBB might promote the apoptosis of cervical cancer cells. Our observation that UBB interacts with HSPC238 implies that UBB may be involved in the multi-step processes of tumorigenesis through the ubiquitin-proteasome pathway (Fig. 5).

In summary, the four proteins screened in the present study have both direct and indirect relationships with the ubiquitin-proteasome pathway. The dysregulation of the ubiquitin-proteasome pathway is closely associated with the development of a variety of diseases, including diseases of the cardiovascular system (19-21), lymphoma (22), kidney disease $(23,24)$, nervous system disorders $(25,26)$, gastrointestinal malignancies (27-29) and has an especially strong association with the development of many cancers. HSPC238 has a RING-finger domain and E3 enzyme activity. Since E3 enzyme activity is a key component of the ubiquitin-proteasome pathway, we speculate that HSPC 238 may interact with HO-1, MT2A, RPS27a and UBB in the ubiquitin-proteasome pathway. Thus, HSPC238 may suppress tumorigenesis by: i) indirectly promoting the ubiquitination of the cancer-promoting protein RPS27a; ii) inhibiting the NF- $\kappa$ B pathway through the binding of $\mathrm{Zn}^{2+}$ and the formation of a complex with MT2A; iii) preventing the ubiquitination of tumor suppressor protein HO- 1 by binding $\mathrm{Zn}^{2+}$; and iv) combining and regulating the function of the small molecule ubiquitin UBB.

\section{Acknowledgements}

This research was supported by the Natural Science Foundation of China (no. 81101534); the Natural Science Foundation of Guangdong Province (no. S2012010010824); and the Medical Research Foundation of Guangdong Province (no. A2013875).

\section{References}

1. Joazeiro CA, Wing SS, Huang H, Leverson JD, Hunter T and Liu YC: The tyrosine kinase negative regulator $\mathrm{c}-\mathrm{Cbl}$ as a RING-type, E2-dependent ubiquitin-protein ligase. Science 286: 309-312, 1999.

2. Brophy TM, Raab M, Daxecker H, Culligan KG, Lehmann I, Chubb AJ, Treumann A and Moran N: RN181, a novel ubiquitin E3 ligase that interacts with the KVGFFKR motif of platelet integrin alpha(IIb)beta3. Biochem Biophys Res Commun 369: 1088-1093, 2008

3. Wang S, Huang X, Li Y, Lao H, Zhang Y, Dong H, Xu W, Li JL and Li M: RN181 suppresses hepatocellular carcinoma growth by inhibition of the ERK/MAPK pathway. Hepatology 53: 1932-1942, 2011

4. Lange R, Christoph A, Thiesen HJ, Vopper G, Johnson KR, Lemaire L, Plomann M, Cremer H, Barthels D and Heinlein UA: Developmentally regulated mouse gene NK10 encodes a zinc finger repressor protein with differential DNA-binding domains. DNA Cell Biol 14: 971-981, 1995.

5. Wong JM, Mafune K, Yow H, Rivers EN, Ravikumar TS, Steele GD Jr and Chen LB: Ubiquitin-ribosomal protein S27a gene overexpressed in human colorectal carcinoma is an early growth response gene. Cancer Res 53: 1916-1920, 1993.

6. Ganger DR, Hamilton PD, Klos DJ, Jakate S, McChesney L and Fernandez-Pol JA: Differential expression of metallopanstimulin/S27 ribosomal protein in hepatic regeneration and neoplasia. Cancer Detect Prev 25: 231-236, 2001.

7. Fatima G, Mathan G and Kumar V: The HBx protein of hepatitis $B$ virus regulates the expression, intracellular distribution and functions of ribosomal protein S27a. J Gen Virol 93: 706-715, 2012.

8. Wu ML, Layne MD and Yet SF: Heme oxygenase-1 in environmental toxin-induced lung disease. Toxicol Mech Methods 22: 323-329, 2012.

9. Caballero F, Meiss R, Gimenez A, Batlle A and Vazquez E: Immunohistochemical analysis of heme oxygenase-1 in preneoplastic and neoplastic lesions during chemical hepatocarcinogenesis. Int J Exp Pathol 85: 213-222, 2004.

10. Zou C, Zhang H, Li Q, Xiao H, Yu L, Ke S, Zhou L, Liu W, Wang W, Huang H, et al: Heme oxygenase-1: a molecular brake on hepatocellular carcinoma cell migration. Carcinogenesis 32 : 1840-1848, 2011.

11. Lin PH, Chiang MT and Chau LY: Ubiquitin-proteasome system mediates heme oxygenase-1 degradation through endoplasmic reticulum-associated degradation pathway. Biochim Biophys Acta 1783: 1826-1834, 2008.

12. Coyle P, Philcox JC, Carey LC and Rofe AM: Metallothionein: the multipurpose protein. Cell Mol Life Sci 59: 627-647, 2002.

13. Tao X, Zheng JM, Xu AM, Chen XF and Zhang SH: Downregulated expression of metallothionein and its clinicopathological significance in hepatocellular carcinoma. Hepatol Res 37: 820-827, 2007.

14. Mao J, Yu H, Wang C, Sun L, Jiang W, Zhang P, Xiao Q, Han D, Saiyin H, Zhu J, et al: Metallothionein MT1M is a tumor suppressor of human hepatocellular carcinomas. Carcinogenesis 33: 2568-2577, 2012.

15. Pan Y, Huang J, Xing R, Yin X, Cui J, Li W, Yu J and Lu Y: Metallothionein 2A inhibits NF-kappaB pathway activation and predicts clinical outcome segregated with TNM stage in gastric cancer patients following radical resection. J Transl Med 11: 173, 2013.

16. Habel N, Hamidouche Z, Girault I, Patiño-García A, Lecanda F, Marie PJ and Fromigué O: Zinc chelation: a metallothionein 2A's mechanism of action involved in osteosarcoma cell death and chemotherapy resistance. Cell Death Dis 4: e874, 2013.

17. Bao B, Prasad AS, Beck FW and Sarkar FH: Zinc up-regulates NF-kappaB activation via phosphorylation of IkappaB in HUT-78 (Th0) cells. FEBS Lett 581: 4507-4511, 2007. 
18. Tian Y, Ding W, Wang Y, Ji T, Sun S, Mo Q, Chen P, Fang Y, Liu J and Wang B, et al: Ubiquitin B in cervical cancer: critical for the maintenance of cancer stem-like cell characters. PLoS One 8: e84457, 2013.

19. Cacciapuoti F: Role of ubiquitin-proteasome system (UPS) in left ventricular hypertrophy (LVH). Am J Cardiovasc Dis 4: 1-5, 2014.

20. Powell SR, Herrmann J, Lerman A, Patterson C and Wang X: The ubiquitin-proteasome system and cardiovascular disease. Prog Mol Biol Transl Sci 109: 295-346, 2012.

21. Schlossarek S and Carrier L: The ubiquitin-proteasome system in cardiomyopathies. Curr Opin Cardiol 26: 190-195, 2011.

22. Suh KS, Tanaka T, Sarojini S, Nightingale G, Gharbaran R, Pecora A and Goy A: The role of the ubiquitin proteasome system in lymphoma. Crit Rev Oncol Hematol 87: 306-322, 2013

23. Fukasawa $\mathrm{H}$ : The role of the ubiquitin-proteasome system in kidney diseases. Clin Exp Nephrol 16: 507-517, 2012.

24. Thomas SS and Mitch WE: Mechanisms stimulating muscle wasting in chronic kidney disease: the roles of the ubiquitinproteasome system and myostatin. Clin Exp Nephrol 17: 174-182, 2013.
25. Rogers N, Paine S, Bedford L and Layfield R: Review: the ubiquitin-proteasome system: contributions to cell death or survival in neurodegeneration. Neuropathol Appl Neurobiol 36: 113-124, 2010.

26. Ying $\mathrm{Z}$, Wang $\mathrm{H}$ and Wang G: The ubiquitin proteasome system as a potential target for the treatment of neurodegenerative diseases. Curr Pharm Des 19: 3305-3314, 2013.

27. Dawson SP: Hepatocellular carcinoma and the ubiquitin-proteasome system. Biochim Biophys Acta 1782: 775-784, 2008.

28. Grande E, Earl J, Fuentes R and Carrato A: New targeted approaches against the ubiquitin-proteasome system in gastrointestinal malignancies. Expert Rev Anticancer Ther 12: 457-467, 2012.

29. Voutsadakis IA: The ubiquitin-proteasome system in colorectal cancer. Biochim Biophys Acta 1782: 800-808, 2008. 